\title{
The frequency of stellar X-ray flares from a large-scale XMM-Newton sample
}

\author{
John P. Pye and Simon R. Rosen \\ University of Leicester, Dept. of Physics \& Astronomy, \\ Leicester, LE1 7RH, U.K. \\ email: pye@le.ac.uk ; srr11@le.ac.uk
}

\begin{abstract}
We present estimates of cool-star X-ray flare rates determined from the XMM-Tycho survey (Pye et al. 2015, A\&A, 581, A28), and compare them with previously published values for the Sun and for other stellar EUV and white-light samples. We demonstrate the importance of applying appropriate corrections, especially in regard to the total, effective size of the stellar sample. Our results are broadly consistent with rates reported in the literature for Kepler white-light flares from solar-type stars, and with extrapolations of solar flare rates, indicating the potential of stellar X-ray flare observations to address issues such as 'space weather' in exoplanetary systems and our own solar system.
\end{abstract}

Keywords. X-rays: stars - stars: flare - stars: activity - stars: coronae - surveys - catalogs

\section{Introduction}

The XMM-Newton Serendipitous Source Catalogue (Watson et al. 2009) has been used as the basis for a uniform, large-scale survey of X-ray flares from late-type (i.e. spectral type F-M) stars in the Hipparcos Tycho catalogue (Høg et al. 2000), as reported by Pye et al. (2015). The XMM catalogue and its associated data products provide an excellent basis for a comprehensive and sensitive survey of stellar flares - both from targeted active stars and from those observed serendipitously in the half-degree diameter field-of-view of each observation. Our sample contains $\sim 130$ flares with well-observed profiles; they originate from $\sim 70$ stars. The flares range in duration from $\sim 10^{3}$ to $\sim 10^{4} \mathrm{~s}$, have peak X-ray fluxes from $\sim 10^{-13}$ to $\sim 10^{-11} \mathrm{erg} \mathrm{cm}^{-2} \mathrm{~s}^{-1}$, peak X-ray luminosities from $\sim 10^{29}$ to $\sim 10^{32} \mathrm{erg} \mathrm{s}^{-1}$, and X-ray energy output from $\sim 10^{32}$ to $\sim 10^{35} \mathrm{erg}$. Most of the 36 flaring, serendipitously-observed stars have little previously reported information, though $\sim 70 \%$ of them have assigned spectral types (mostly $\mathrm{F}-\mathrm{K}$, with a few $\mathrm{M}$ ). The total number of serendipitously-observed Tycho stars with 2XMM light-curves (i.e. ones for which we could potentially detect flaring) was $\sim 500$.

In this paper, we focus on and extend one specific aspect of the work reported by Pye et al. (2015), namely the rate of flaring as derived from the serendipitous sample, and comparison with other stellar and solar results reported in the literature.

\section{Flare rates and frequency distributions}

The serendipitous observations provide an unbiased (with respect to stellar activity) study of flare energetics. The serendipitous sample demonstrates the need for care when calculating flaring rates, especially when normalising the number of flares to a total exposure time, where it is important to consider both the stars seen to flare and those measured as non-variable, since in our survey, the latter outnumber the former by more than a factor ten. Both sets of stars appear very similar in terms of the distributions of 


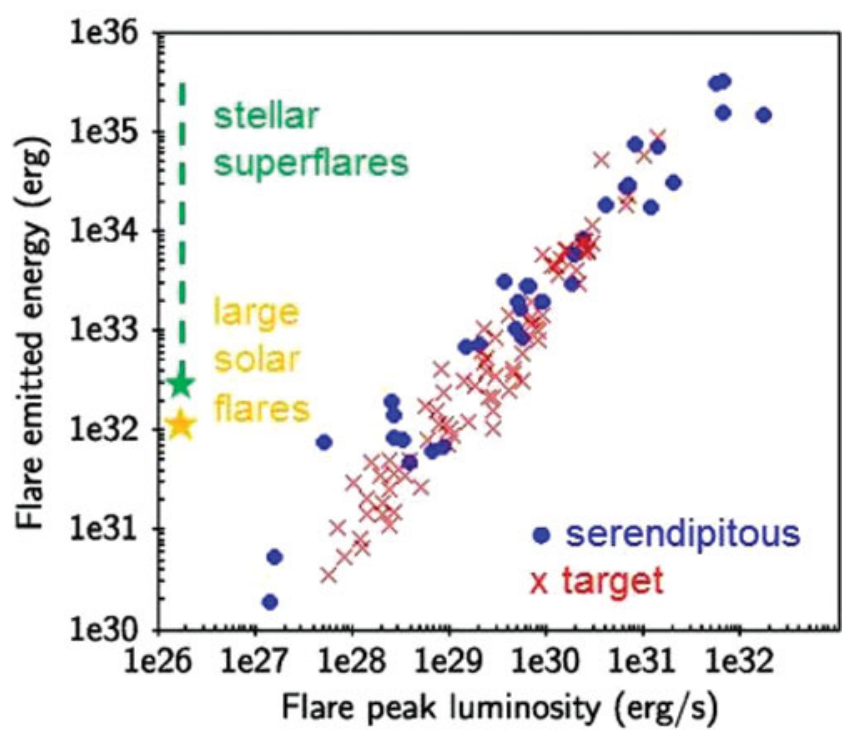

Figure 1. Total X-ray emitted energy $E_{\mathrm{X}}$ versus flare peak X-ray luminosity (energy band 0.2 - $12 \mathrm{keV}$ ) (after Pye et al. 2015). Only 'fully-observed' flares are shown (see Pye et al. 2015 for details). Key to symbols: blue circles: Serendipitously-observed stars; red diagonal crosses: Target stars. Nominal values of $E_{\mathrm{X}}$ for large solar flares (yellow star symbol) and stellar superflares (green star symbol and dashed line) are indicated.

general properties such as quiescent X-ray luminosity. It may well be that the lack of observed flaring arises simply from a combination of the relatively limited observation time for each star and the range of activity levels exhibited by cool stars in general (Pye et al. 2015).

\subsection{Comparison with solar and other stellar results}

Fig. 1 shows the total X-ray emitted energy $\left(E_{\mathrm{X}}\right)$ versus flare peak X-ray luminosity $\left(L_{\mathrm{X} \text {,peak }}\right)$, for our serendipitous and target samples, together with an indication of the regions occupied by large solar flares and stellar superflares (see e.g. Güdel 2004 ; Schrijver et al. 2012; Shibayama et al. 2013), showing that many of the XMM-Tycho flares come within the 'superflare' category, and that there is substantial overlap in terms of energetics between the observed stellar flares and large solar flares.

We have constructed frequency distributions for $E_{\mathrm{X}}$ and derived flare rates above specified thresholds (Pye et al. 2015). Due to the small numbers of stars, and the lack of detailed information for most of the serendipitous sample, we have not attempted to divide them into different categories; hence the distributions and statistics refer to a rather heterogeneous collection of stellar types. We also note that there are large uncertainties due to the relatively small number of flares observed, and there may be incompleteness effects, as discussed by Pye et al. (2015).

In Fig. 2, our results are compared with published values for solar flares and for other stellar surveys. The latter comprise EUV observations of several known, active stars (Audard et al. 2000), and white-light stellar flares from solar-type stars observed by the Kepler mission (e.g. Maehara et al. 2015). Note that Fig. 2 represents bolometric energy $\left(E_{\mathrm{bol}}\right)$; we have converted our X-ray values using $E_{\mathrm{bol}} / E_{\mathrm{X}}=4$, i.e. within the range $E_{\mathrm{bol}} / E_{\mathrm{X}} \sim 3-5$ suggested by Schrijver et al. (2012).

We summarise our findings and comparisons as follows.

- Previously reported (e.g. Schrijver et al. 2012) comparisons of solar-flare frequency 


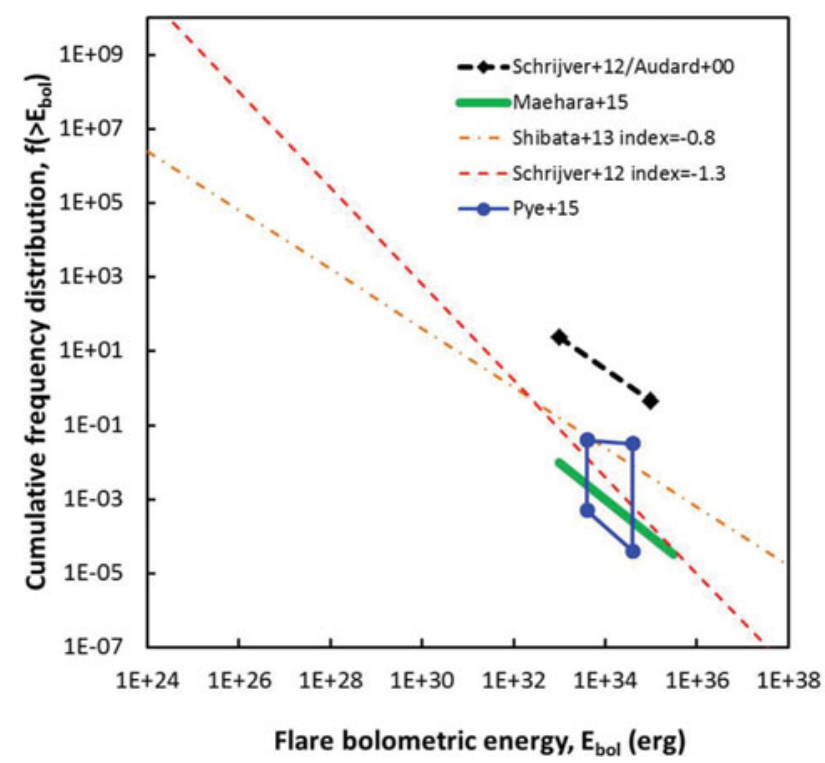

Figure 2. Comparison of various solar- and stellar-flare cumulative frequency distributions, $f\left(>E_{\mathrm{bol}}\right)$ (number per year per star) in terms of bolometric energy, $E_{\mathrm{bol}}(\mathrm{erg})$. Key to symbols: blue solid lines forming a trapezium, with circle symbols: X-ray (this work; Pye et al. 2015), lower pair of points are scaled according to stellar coronal quiescent luminosity, upper pair are not scaled; thick green solid line: Kepler white-light results (Maehara et al. 2015); thick black dashed line with diamond symbols: EUV results scaled according to stellar coronal quiescent luminosity (after Audard et al. 2000; Schrijver et al. 2012); red dashed line: power-law with index -1.3, representing solar results and extrapolation to high energies (Schrijver et al. 2012); orange dash-dot line: power-law with index -0.8 , representing solar results and extrapolation to high energies (Shibata et al. 2013).

distributions (albeit somewhat extrapolated) with scaled (downwards) EUV distributions for several highly-active stars (from Audard et al. 2000) have suggested that the Sun appears to lie significantly below other stars, by a factor $\sim 100$, even after allowing for the differences in overall, 'quiescent' coronal (EUV/X-ray) luminosity. Our results (see also Shibayama et al. 2013) indicate that this is likely to be due to the bias from having only very active stars in the EUV sample.

- Our scaled XMM-Tycho flare rates (for $E_{\mathrm{X}}>10^{33} \mathrm{erg}$ ) are:

○ a factor $\sim 2 \times 10^{4}$ lower than the highly-active-star EUV scaled rates;

o broadly consistent with extrapolated solar rates (Schrijver et al. 2012; Shibayama et al. 2013);

o broadly consistent with Kepler white-light rates (e.g. Shibayama et al. 2013; Maehara et al. 2015).

\section{Future work}

We summarise here several obvious areas in which this work may be carried forward. Our aim would be to have a survey with well-characterised stellar properties and of sufficient size to enable, for example, estimation of flare rates for solar-type stars (c.f. the visible-light results, see e.g. the review by Shibata, this volume).

- Detailed characterisation of the stars, via follow-up at optical wavelengths: spectroscopy to determine whether a star is single or comprises a close binary system, and 
to measure rotation velocity $(v \sin i)$ and, where applicable, orbital period; photometry (typically over durations of $\sim$ weeks to $\sim$ months), to determine rotation period.

- Modelling the 3-dimensional spatial distribution of the stars, to enable better correction for incompleteness effects.

- Extension of the X-ray observations, by taking account of the factor $\sim 3$ increase in data now available from the 3XMM catalogue (Rosen et al. 2016) and utilising the timevariability data products and characterisation to be produced by the EU-FP7 EXTraS project ( http://www.extras-fp7.eu/ ; De Luca et al. 2015; Pizzocaro et al. 2015).

- Extension of the stellar catalogues, especially to expand the coverage of dM-type stars.

In the long-term, wide-field X-ray surveys, e.g. with 'Lobster-eye' optics (e.g. Osborne et al. 2013), may be expected to yield many observations of stellar flares.

\section{Conclusions}

We have shown broad consistency between our X-ray flare rates and those extrapolated and scaled from the Sun and from stellar white-light observations. There are a number of possible future activities which would carry this work forward in terms of larger sample sizes and greater knowledge of the stellar properties, thus improving our insights into both the mechanisms for flare generation, and the 'space weather' effects of flares on the stellar-system environment and any exoplanets present.

\section{References}

Audard, M., Güdel, M., Drake, J. J., \& Kashyap, V. L. 2000, ApJ, 541, 396

De Luca, A., Salvaterra, R., \& Tiengo, A., et al. 2015, in: Longo, Napolitano, Marconi, Paolillo \& Iodice (eds.), The Universe of Digital Sky Surveys, to be published in ApESSSP, arXiv: 1508.07146

Güdel, M. 2004, A\&AR, 12, 71

Høg, E., Fabricius, C., Makarov, V. V., et al. 2000, A\&A, 355, L27

Maehara, H., Shibayama, T., \& Notsu, Y., et al. 2015, Earth, Planets \& Space, 67, 59

Osborne, J. P., O'Brien, P., Evans, P., et al. 2013, in: A. J. Castro-Tirado, J. Gorosabel, \& I. H. Park (eds.), EAS Publications Series, Vol. 61, 625

Pizzocaro, D., Stelzer, B., \& Paladini, A., et al. 2015, submitted to A\&\&A

Pye, J. P., Rosen, S., Fyfe, D., \& Schröder, A. C. 2015, A\&A, 581, A28

Rosen, S. R., Webb, N. A., \& Watson, M. G., et al. 2016, A\&A, 590, A1

Schrijver, C. J., Beer, J., Baltensperger, U., et al. 2012, J. Geophys. Res. (Space Physics), 117, 8103

Shibata, K., Isobe, H., \& Hillier, A., et al. 2013, PASJ, 65, 49

Shibayama, T., Maehara, H., \& Notsu, S., et al. 2013, ApJS, 209, 5

Watson, M. G., Schröder, A. C., \& Fyfe, D., et al. 2009, A\&AA, 493, 339 